\title{
Use of Clinoptilolite in Ethanol Dehydration
}

\author{
FUNDA TIHMILLIOGLU \\ CHEMICAL ENGINEERING DEPARTMENT \\ EGE UNIVERSITY \\ BORNOVA, IZMIR, TURKEY
}

SEMRA ULKU*

IZMIR INSTITUTE OF TECHNOLOGY

BASMANE, IZMIR, TURKEY

\begin{abstract}
Clinoptilolite-type natural zeolite, which exists in various regions of Turkey, has been experimentally studied. For the ethanol-water-local clinoptilolite system, uptake and breakthrough curves were determined under a nitrogen gas atmosphere. In adsorption kinetics and adsorption equilibrium studies, the effects of particle size, temperature and, amount of zeolite on the uptake rate have been investigated. The breakthrough curves for four different flow rates of ethanol and three different bed heights were determined in dynamic column studies. The results of the experiments show that intraparticle diffusion is the main resistance. The local clinoptilolite is a promising adsorbent for water adsorption from aqueous ethanol.
\end{abstract}

\section{INTRODUCTION}

Adsorption technologies have recently attracted interest due to their potential application in bulk gas and liquid mixture separations and commercial trace component purification. Since adsorption separation and purification processes often provide the least energy-consuming route, they are becoming common in chemical and petrochemical industries as an alternative process to distillation which is not energy efficient.

* To whom correspondence should be addressed: Gaziosmanpaşa Bulvari No. 16, 35230 Çankaya, Turkey. 
Availability of suitable adsorbents with economical prices is the essential key for the success of adsorption separation technology. Activated carbon, zeolite, silica gel, activated alumina, and activated clay are the common commercially available adsorbents. Although activated carbon, silica gel, activated alumina, and activate clay have nonuniform pore sizes (with a wide distribution of pore sizes within the adsorbent particle), zeolite molecular sieves have pore sizes $(\approx 0.3-1 \mathrm{~nm})$ which are almost uniform throughout the particle. For all the adsorbents except zeolites adsorption depends greatly on the fluid phase concentration, and is very low at low concentrations. These properties of the zeolites make them especially convenient for selective separation and purification processes.

Zeolites are crystalline hydrated alumina silicates of Group I and II elements. There are more than 150 synthetic and more than 40 natural different types. Although the use of synthetic zeolites is becoming common in chemical and petrochemical industries, the natural ones have found limited commercial application. Clinoptilolite, a silica-rich natural zeolite with a bidisperse pore size distribution, has been used commercially as a selective gas or vapor adsorbent and ion exchanger in several countries. It has some special value for moisture removal. Clinoptilolite is found in sedimentary rocks in various parts of Turkey, especially in the Western part of Anatolia, as an alternative product of volcanic derivatives. Ulku and colleagues conducted several studies on the utilization of this local mineral as an adsorbent for water vapor, especially in drying and energy recovery systems, and compared the water adsorption value of this local mineral with other desiccant materials (1-6).

The zeolites and the other adsorbents are generally used in the form of pellets prepared from microporous crystals with suitable binders. The binder affects the adsorption process by altering the pore structure of the absorbent material through introducing mesopores and macropores. When these pellets are packed in a bed, the voids between these pellets become also effective in the process. Although intraparticle adsorbate transfer is generally the rate-limiting step and Fick's law (with proper concentration gradients and diffusivities) is used in the description of the possible mechanisms (macropore diffusion, surface diffusion, micropore diffusion), heat transfer may also be effective due to the exothermic nature of the process. Pore structure of the adsorbent, properties of the adsorbent and adsorptive, and process variables (temperature, pressure, concentration range) specify the controlling mechanisms.

The production of absolute alcohol from aqueous solution requires almost complete removal of the residual moisture. The cost of separation and purification is one of the major considerations in absolute alcohol production. The conventional process of separation is by distillation. Be- 
cause of the formation of a minimum boiling point azeotrope, distillation yields only $95 \mathrm{wt} \%$ ethanol under normal conditions. Further purification to produce anhydrous ethanol is normally accomplished by azeotropic distillation using a volatile entrainer or extractive distillation employing an extractive solvent. The high costs of these energy-intensive processes have spurred research to develop other nonconventional processes.

The adsorption dehydration of ethanol solutions with synthetic zeolites has attracted attention in recent years. Teo and Chen (7) examined the liquid-phase dehydration of aqueous ethanol using zeolites $3 \mathrm{~A}$ and $4 \mathrm{~A}$ in adsorption columns under ambient conditions. They applied the dual resistance model of Weber and Chakravorti (8) which considers intraparticle diffusion and external film resistances, and thereby obtained better prediction of the breakthrough curves in the initial region. In the experimental study of Sowerby and Crittenden $(9,10)$, the recovery of ethanol from aqueous vapors was investigated for columns packed with pellets of various synthetic zeolites; the adsorption capacity of $4 \mathrm{~A}$ zeolite was found to be highest. In the studies of Teo and Ruthven (11), the uptake curves for a closed batch system and the breakthrough curves for a jacketed glass column with $3 \mathrm{~A}$ zeolite as adsorbent were determined. It was shown that the uptake rate is controlled primarily by intraparticle pore diffusion resistance with some additional contribution from the external film resistance. In the studies of Colella and colleagues (12), phillipsite rich volcanic tuffs were proposed for the dehydration of ethanol vapors, and preliminary studies on the water adsorption kinetics and column dynamics were described.

\section{EXPERIMENTAL}

Due to the affinity of the alcohol and the zeolite for water, the sampling, the transfer, and the storage of the materials were quite critical. Special care was necessary to prevent the adsorption of moisture from the environment. Applications of evacuation and of a dry nitrogen environment were very helpful to the reliability of the experiments and the accuracy of the results.

A natural zeolite, local clinoptilolite from Bigadic, Turkey, in a 1.5-3 $\mathrm{mm}$ particle size was used as the adsorbent. Some tests were also conducted with the synthetic zeolite $4 \mathrm{~A}$ for comparison. The zeolite samples were preconditioned by thermal activation in a furnace at $300^{\circ} \mathrm{C}$ for 24 hours and then stored in a vacuum desiccator before use in adsorption studies.

Ethanol-water mixtures in the range of 2.2-6.6 wt \% water were prepared by using distilled water and analytical grade absolute alcohol $(0.79$ 
g/L, minimum purity $99.8 \mathrm{wt} \%$ ) supplied by Merck. Their concentrations were determined by gas chromatography (Hewlett-Packard Model 19001A-101 HP with a Chromosorb-101 column and a thermal conductivity detector). All sampling was conducted under a dry nitrogen gas atmosphere. After evacuation of the lines, the samples were collected in previously evacuated sample holders and sealed immediately.

\section{Batch Kinetic Experiments}

In this group of experiments the effects of the zeolite amount, the particle size, and the temperature on the adsorption kinetics and equilibrium were investigated. $100 \mathrm{~mL}$ of aqueous ethanol (6.6 wt\% water) and a known amount of the zeolite particles were put into the closed system which was initially evacuated and kept under nitrogen gas atmosphere in a constant temperature bath. Samples from the liquid phase were taken against time, and the rates of water uptake by the adsorbent from the ethanol-water mixtures were monitored over ranges of the zeolite amount $(10-50 \mathrm{~g})$, the bath temperature $\left(15-35^{\circ} \mathrm{C}\right)$, and the particle size $(1.5-3.8$ $\mathrm{mm}$ ). The equilibrium liquid-phase concentrations were obtained by allowing 4 days for the adsorbent-liquid mixtures to come to equilibrium.

\section{Dynamir Column Experiments}

Breakthrough experiments for the dehydration of aqueous ethanol were carried out in a glass column $(3.7 \mathrm{~cm} \times 1 \mathrm{~m}$ height $)$ packed with the preconditioned clinoptilolite under a nitrogen gas atmosphere. The feed, an ethanol-water mixture containing about $6.3 \mathrm{wt} \%$ water, was introduced from the top. Experiments were performed for four different flow rates ranging from 5.5 to $15 \mathrm{~mL} / \mathrm{min}$ and for three bed heights $(45,75$, and 90 $\mathrm{cm})$. Samples of the effluent from the column were collected at suitable time intervals and analyzed chromatographically for the water and ethanol contents.

\section{RESULTS}

The adsorbed phase concentrations were determined by mass balances by knowing the liquid-phase concentrations. The changes in the liquidphase concentrations with time for different initial concentrations are given in Fig. 1. For the adsorption of water on the clinoptilolite, a highly favorable isotherm which approaches a rectangular or irreversible form, shown in Fig. 2, was obtained. The adsorption isotherm of $4 \mathrm{~A}$ synthetic zeolite is also shown in Fig. 2 for comparison. 


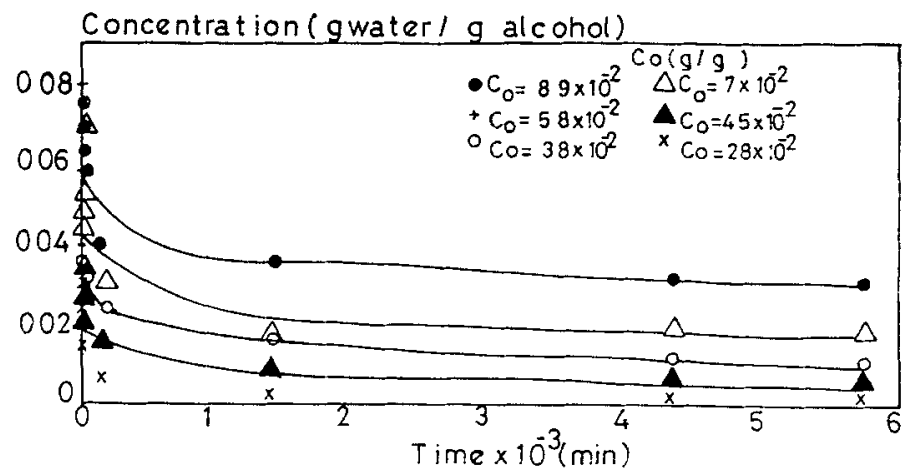

FIG. 1 The change of water concentration in aqueous ethanol with time.

\section{Analysis of Batch Uptake Experiment Results}

Some representative uptake curves showing the effects of the zeolite amount, the particle size, and the bath temperature for rectified alcohol drying are presented in Figs. 3, 4, 5, and 6. In most of the experiments the final uptake of the sorbate (adsorption capacity of the clinoptilolite) was about $0.13 \mathrm{~g}$ water/g dry zeolite, but slightly lower values were obtained in some experiments. This discrepancy is mainly due to the imperfect dehydration, storage, and transfer conditions of the adsorbent. Within the experimental range of studies $(20-50 \mathrm{~g})$, the greater the amount of the

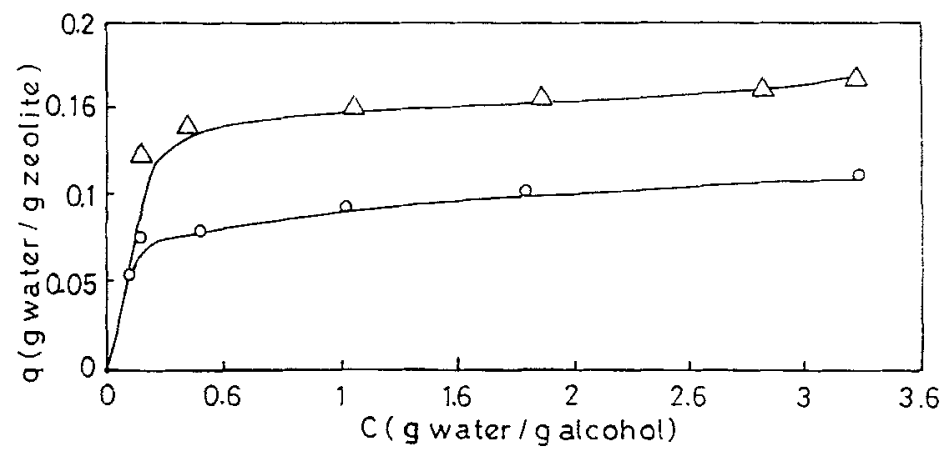

FIG. 2 Comparison of the adsorption isotherms of local clinoptilolite $(O)$ and synthetic zeolite $4 A(\triangle)$. 


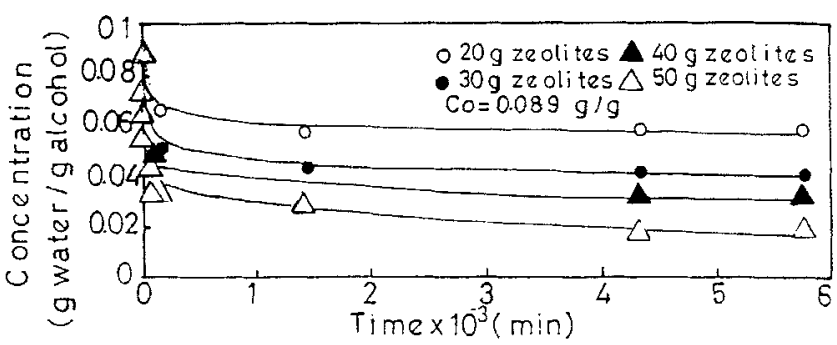

FIG. 3 Effect of zeolite amount on concentration.

zeolite used, the lower the water concentration attained in the solution for the same adsorption period, as expected.

Investigation of the effect of temperature showed that decreasing the bath temperature increased the adsorption capacity for similar operational conditions, so within the experimental range of studies $\left(15-35^{\circ} \mathrm{C}\right)$, the lowest temperature experiments gave the highest ethanol concentration, as expected. Comparison of the effect of particle size on the adsorption capacity of the clinoptilolite indicated a greater adsorption capacity for smaller-sized zeolites for the same adsorption period. A similar behavior was observed in the work of Teo and Ruthven (11) for $3 \mathrm{~A}$ synthetic zeolite.

The transient uptake curve relationship with intraparticle diffusion control under isothermal conditions is

$$
\left(m_{1} / m_{\alpha}\right)=\frac{q-q_{0}}{q_{\alpha}-q_{0}}=1-\left(6 / \pi^{2}\right) \sum_{n=1}^{\alpha}\left(1 / n^{2}\right) \exp \left(-n^{2} \pi^{2} D t / R_{\mathrm{p}}^{2}\right)
$$

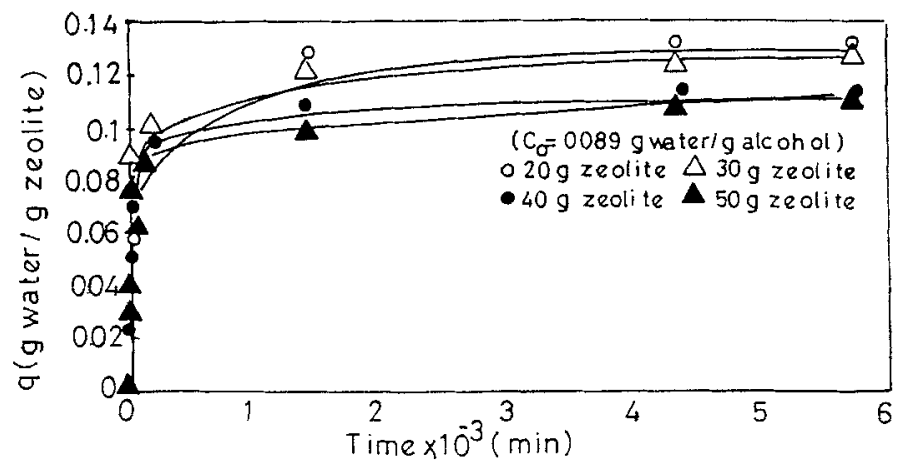

FIG. 4 Zeolite amount and the uptake curves. 


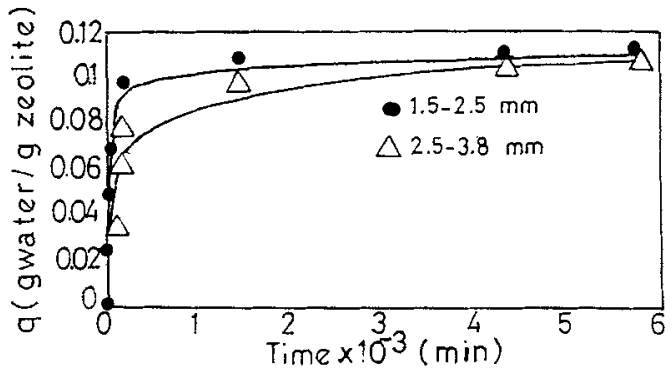

FIG. 5 Particle size and the uptake curves.

It was used for the analysis. The diffusivity of water in the local clinoptilolite was calculated from the uptake curve relationship using the shorttime-region solution:

$$
\frac{m_{\mathrm{t}}}{m_{\alpha}} \cong \frac{6}{\sqrt{\pi}}\left(\frac{D t}{R_{\mathrm{p}}^{2}}\right)^{1 / 2}
$$

The value found was $3 \times 10^{-11} \mathrm{~m} / \mathrm{s}$.

\section{Analysis of Experimental Breakthrough Curves}

The breakthrough curves for water adsorption from aqueous ethanol as determined for different flow rates and bed heights are presented in Figs. 7 and 8. Figure 7 shows that an increase in the feed flow rate decreases the time required to attain the breakthrough point, as expected. Compari-

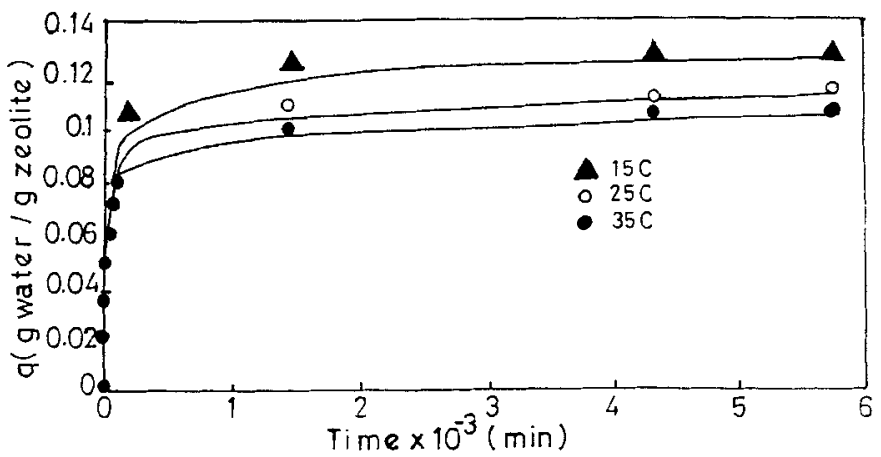

FIG. 6 Effect of temperature on the uptake curves. 


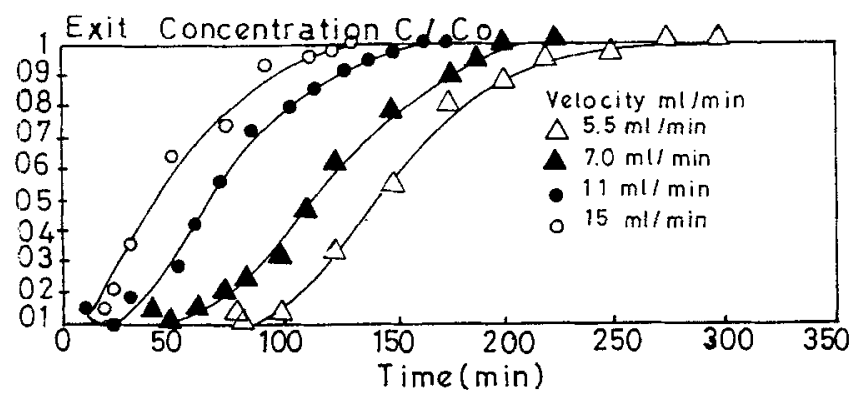

FIG. 7 Effect of feed flow rate on the breakthrough curves.

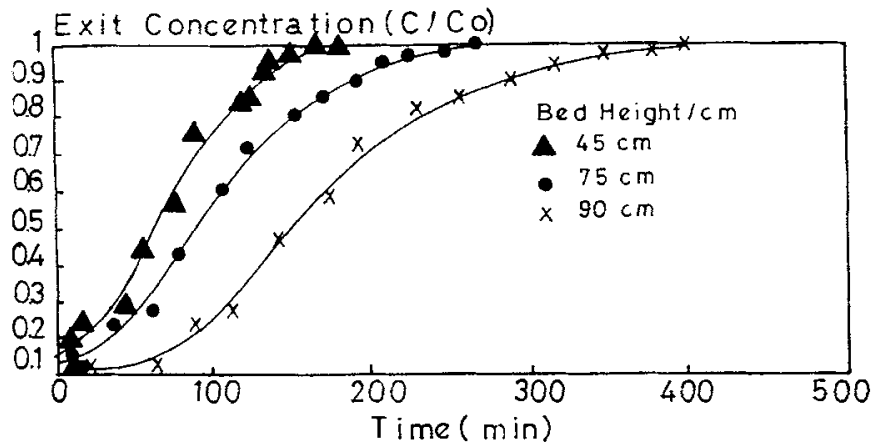

FIG. 8 Effect of packing height on the breakthrough curves.

TABLE 1

Water Adsorption Capacity of Clinoptilolite

\begin{tabular}{lcccccc}
\hline$M(\mathrm{~g})$ & 400 & 400 & 400 & 400 & 550 & 700 \\
$V(\mathrm{~mL} / \mathrm{min})$ & 5.5 & 7.0 & 11.0 & 15.0 & 11.0 & 11.0 \\
$q(\mathrm{~g}$ water/g zeolite $)$ & 0.112 & 0.114 & 0.118 & 0.119 & 0.121 & 0.131 \\
\hline
\end{tabular}

TABLE 2

External Mass Transfer Coefficients and Biot Number

\begin{tabular}{lcccc}
\hline$V(\mathrm{~mL} / \mathrm{min})$ & 5.5 & 7.0 & 11.0 & 15.0 \\
$k_{\mathrm{f}}(\mathrm{cm} / \mathrm{min})$ & 0.027 & 0.033 & 0.047 & 0.06 \\
$\mathrm{Bi}_{\mathrm{m}}$ & 50 & 60 & 87 & 110 \\
\hline
\end{tabular}


sons of the experimental breakthrough curves for different bed heights under otherwise identical conditions confirm a constant behavior pattern of the adsorbent-adsorbate system studied. They also show that an increase in the packing height of the column increases the time required to reach the breakthrough point (Fig. 8).

The adsorption capacities of the clinoptilolite columns were determined from the mass balance for the whole column:

$$
\frac{\rho V C_{0}}{M} \int_{0}^{\mathrm{t}}\left(1-\frac{C}{C_{0}}\right) d t=\int_{0}^{\mathrm{q}} d q
$$

They were compared with the ones obtained from the equilibrium studies. The adsorption capacities of Bigadic clinoptilolite for different experimental conditions are presented in Table 1.

The external mass transfer coefficients were estimated from the correlation of Wakao and Funazkri (13) which provides a good estimation of the mass transfer coefficients in fixed beds over a wide range of Reynolds numbers, as indicated by Ruthven (14):

$$
\mathrm{Sh}=2.0+1.1 \mathrm{Sc}^{0.33} \mathrm{Re}^{0.8}
$$

The relative importance of the external film and intraparticle resistances has been determined from analysis of the mass transfer Biot number for the particle.

Table 2 illustrates Biot numbers for different experimental conditions. The importance of the intraparticle mass transfer resistance relative to the external film resistance is obvious since all the values are considerably higher than 1 (6).

\section{CONCLUSION}

For the same regeneration $\left(300^{\circ} \mathrm{C}, 24\right.$ hours) and operational conditions, water adsorption sapacities of the clinoptilolite-type natural zeolite from Bigadic, Turkey, and 4A synthetic zeolite were found to be 0.13 and 0.165 $\mathrm{g}$ water/g dry zeolite, respectively. For the existing regeneration conditions, which seems to be quite convenient for practical purposes, the difference in the adsorption capacities of the two zeolites is not drastic. Actually, it is possible to increase these capacities by changing the regeneration conditions. The dehydration of aqueous ethanol by the local clinoptilolite seems to have a promising future, but further work is required to upgrade the mineral before it can be used for commercial applications. 


\section{ACKNOWLEDGMENT}

The authors are grateful to Ege University Research Foundation for support of the project.

\section{NOMENCLATURE}

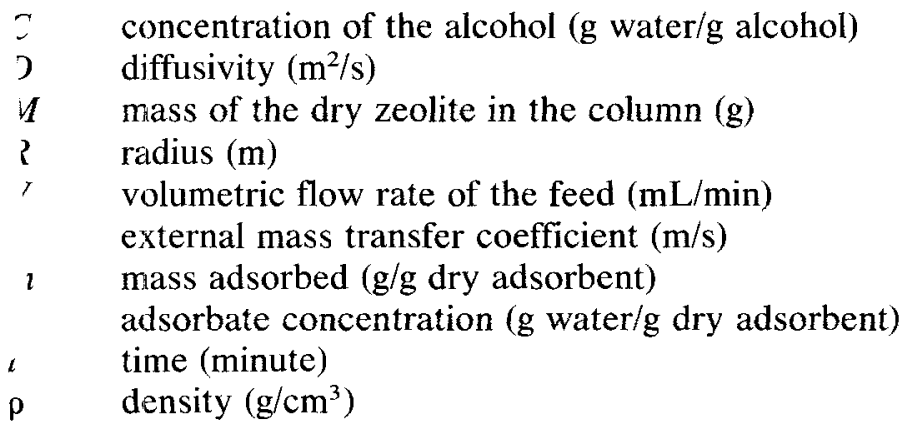

\section{Dimensionless numbers}

$\mathrm{Bi}_{\mathrm{m}} \quad$ Biot number for mass transfer

Re Reynolds number

Sc Schmidt number

Sh Sherwood number

\section{Indices}

f fluid

0 initial, inlet

m molecular

p particle

t at time $t$

$\alpha \quad$ at time $\alpha$

\section{REFERENCES}

1. S. Ulku, "Adsorption Heat Pumps," J. Heat Recovery Syst., 6(4), 277 (1986).

2. S. Ulku and F. Cakicioglu, "Energy Recovery in Drying Applications," Int. J. Renewable Energy, 5, 695 (1991).

3. S. Ulku, “Adsorption Heat Pumps Refrigerators," Solid Sorption Refrigeration Symposium, 1992, p. 86.

4. S. Ulku and M. Mobedi, "Adsorption in Energy Storage," Energy Storage, Vol. 167, Kluwer Academic Publisher, 1989, p. 487. 
5. S. Ulku and M. Mobedi, "Zeolites in Heat Recovery," Studies in Surface Science and Catalysis, Vol. 49, Elsevier Science Publisher, 1989, p. 511.

6. S. Ulku, "Heat and Mass Transfer in Porous Media," Convective Heat and Mass Transfer in Porous Media, Kluwer Academic Publisher, 1991, p. 695.

7. W. K. Teo and T. Chen, "Liquid Phase Dehydration of Ethanol Solution in a Fixed Bed of Molecular Sieves," Appl. Biotechnol., 24(25), 521 (1990).

8. T. W. Weber and R. K. Chakravorti, AIChE J., 20, 228 (1974).

9. B. Sowerby and B. D. Crittenden, "An Experimental Comparison of Type a Molecular Sieves for Drying the Ethanol-Water Azeotrope," Gas Sep. Purif., 2, 77 (1988).

10. B. Sowerby and B. D. Crittenden, "Scale-up Vapour Phase Adsorption Column for Breaking the Ethanol-Water Azeotrope," Ind. Eng. Chem., Process Des. Dev., 118, 5.11-5.15 (1990).

11. W. K. Teo and D. M. Ruthven, "Adsorption of Water from Aqueous Ethanol Using 3A Molecular Sieves," Ibid., 25, 1 (1985).

12. C. Colella, M. Pansini, F. Alfani, M. Cantarella, and A. Gallifuoco, "Selective Water Adsorption from Aqueous Ethanol Containing Vapours by Phillipsite-rich Volcanic Tuffs," Microporous Mater., 3, 219 (1994).

13. N. Wakao and T. Funazkri, Chem. Eng. Sci., 33, 1375 (1978).

14. D. M. Ruthven, Principles of Adsorption and Adsorption Processes, Wiley, New York, NY, 1984.

Received by editor January 22, 1996 